\title{
Application of static tilt adjustment evaluation method in optical recording
}

\author{
Wei Guo ${ }^{1, a}$, Jianghua Zhang ${ }^{2, b}$ \\ ${ }^{1}$ Department of Mechanical Engineering, Dalian Vocational \& Technical College, Dalian, 116037, \\ China \\ ${ }^{2}$ College of Light Industry and Chemical Engineering, Dalian Polytechnic University, Dalian 116034, \\ China \\ aGuostephen@hotmail.com, bzhang_hh@dlpu.edu.cn
}

Keywords: Tilt Adjustment, Evaluation, Optical Recording.

\begin{abstract}
In the optical recording equipment, the angle between the disc and the optical pickup head is the important parameter of the traverse. In this paper, by establish a set of static tilt angle adjusting device, we introduces the static tilt adjustment principle and derive the theoretical formulas.
\end{abstract}

\section{Introduction}

In today's daily electronic data storage, the storage methods include the tape library, the disk array, and optical disc library etc. In recent years, with the development of HD DVD and Blue-ray Disc, the storage capacity of single disc is greatly increased up to 50GB [1,2]. The Optical disc library shows a great advantage than the other methods in performance and price. The optical disc library has been applied as the important storage equipment in many fields, such as bank's bill image storage, document storage of the Insurance institution, and other situations of high capacity off-line information storage. As the most important component, the performance of the mechanism determines the reading and writing quality of the optical disc library. Among many performance parameters of the mechanism, the radial and tangential tilt angles between the emission laser axis of objective lens and test disc are the key parameters to develop, produce and detect the DVD mechanism [3]. At the same time, they are one of the important factors that affect the read and write quality. At the initial stage of development, it is difficult to guarantee the dip angle meeting optical read head to write requirements after the completion of the assembly of the mechanism. Therefore, we improve the application of the tilt adjustment method in the development according to the tilt adjustment theory.

\section{Adjustment theory}

The theory of static tilt adjustment is adjusting the parallel between spindle motor turntable surface and the surface of optical pickup head movement which is composed by Main and Sub shaft to make sure the beam from optical pickup head focusing on the glass disc vertically. This will ensure the offset distance between actual light spot and ideal spot is small enough to read and write the optical disc signal correctly in the working process[4,5].

In the actual adjustment, we use laser emitter to generate the laser simulating that from the optical pickup head, and set up the working system as shown in Figure 1 to adjust and verify the dip angle between the spindle motor turntable surface and the plane composed by the main shaft and the sub shaft. 


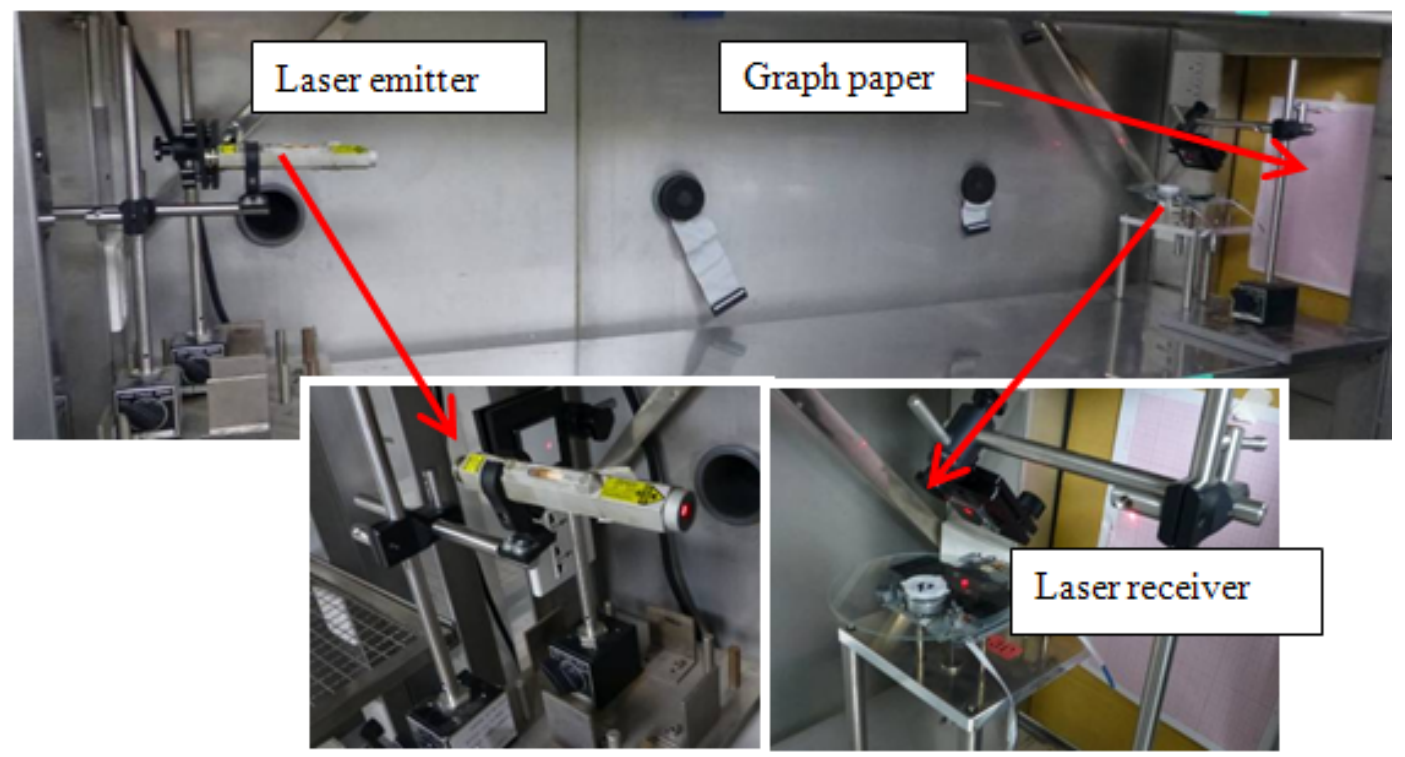

Fig. 1 Work system for tilt adjustment \& measurement

In the operating system, laser emitted from a laser emitter, is reflected in the mirror device (Mirror1) which is set to 45 degree from horizontal, and reached to the rotating glass disc which is under Mirror1, some of these beam reached the Adjust mirror gauge surface which is placed on the main and sub shaft,

The reflected laser from the upper surface of glass disc reverted to Mirror1 and then reached the other mirror (Mirror2) which is set besides Laser emitter. This laser beam is reflected again by Mirror2 and finally reached the graph paper and shows as a rotating light circle (a). The diameter of this circle indicates the verticality of Turntable surface and spindle motor shaft. The bigger the circle diameter is, the worse the verticality is, and the difficulty of signal playback is. As said before, there is some laser beam pass through glass disc and reach the surface of Adjust Mirror Gauge. The gauge's upper surface is parallel to shaft composed with the main and sub shaft. With a excellent precision, we consider parallelism between these two surface is 0 degree.

Due to the parallelism between the glass disc surface (same surface as spindle motor disc placed surface) and the surface composed by main and sub shaft is not 0 degree, the incident laser beam to the Adjust Mirror Gauge produced an angle with the perpendicular line of surface of the Adjust Mirror Gauge.

When the incident laser beam is reflected back through the glass disc and the reflected laser beam go through the same path through like those beams which reflected from the surface of the glass disc. After 2 times reflection, the beam also forms a light spot (b) in the graph paper. This light spot (b) is clear and single because Adjust Mirror Gauge has a smooth surface and do not rotate together with spindle motor.

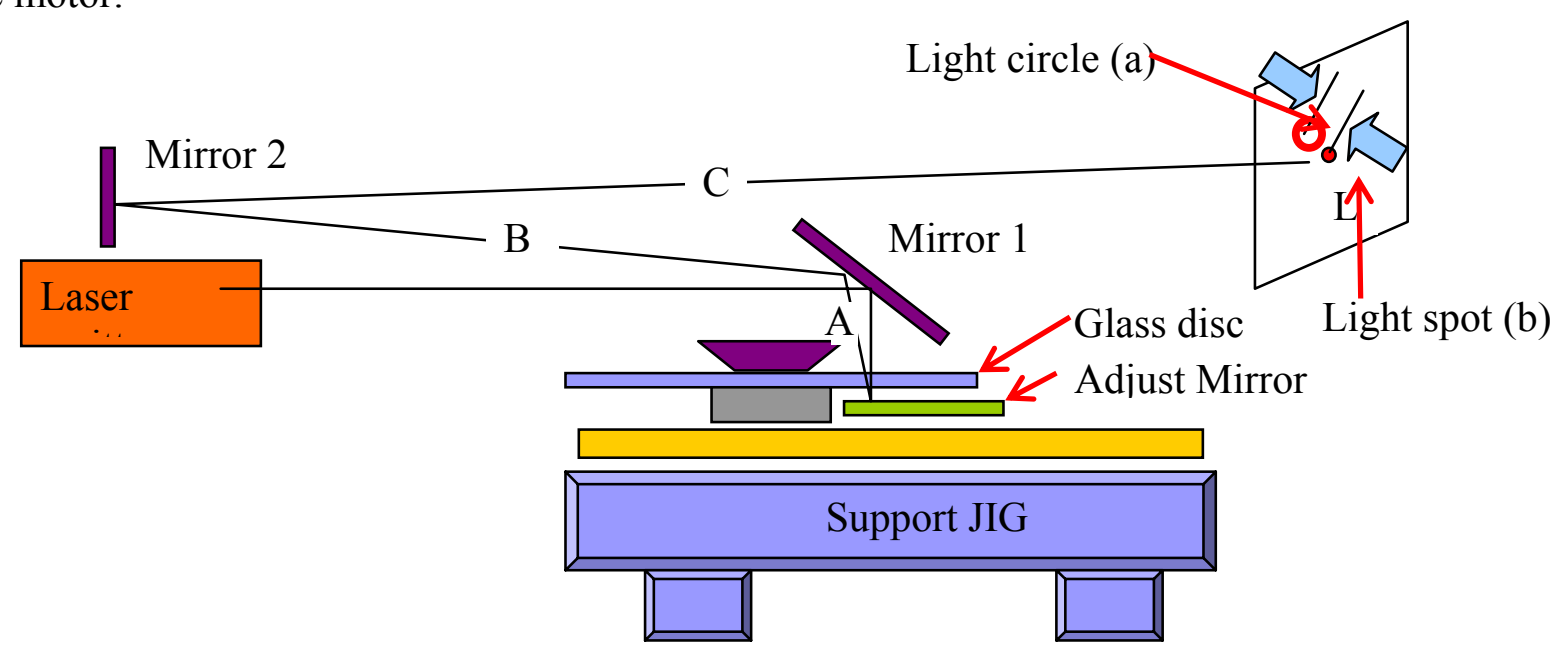

Fig. 2 Illustrative diagram of tilt adjustment \& measurement 
In the practical application of the mechanism development, in order to realize the optimization of optical pickup head's signal pickup, it is requested that laser beam of optical pickup head incident perpendicular to the disc surface, which also requires the parallel between the surface composed by main and sub shaft and spindle motor turntable surface, which means to adjust the light spot $b$ to the center of light circle a.

To study the parallel between the turntable surface and the surface composed by the main and sub shaft is to study the angle between the 2 surfaces. We give out the calculation formulas of the angle as follows according to Fig.2:

$$
\begin{gathered}
A+B+C=D(\mathrm{~m}) \\
\operatorname{Tan}^{-1}((L / 2) / D) * 1000 \approx((L / 2) / D) * 1000=E(\mathrm{mrad}) \\
1 \mathrm{mrad}=0.0573 \mathrm{deg} \quad(180 \mathrm{deg}=1 \pi \mathrm{rad}) \\
E(\mathrm{mrad}) \times 0.0573(\mathrm{deg} / \mathrm{mrad})=F(\mathrm{deg})
\end{gathered}
$$

\section{Adjustment accuracy analysis:}

1. Considered the graph paper lattice distance is $1 \mathrm{~mm}$, the spot $1 \mathrm{~mm}$ change accuracy deviation investigation and substituted into the Eq.2, Eq.4, we can calculate that the angle change is $0.0036 \mathrm{deg}$ when there is a deviation of $1 \mathrm{~mm}$ in graph paper with a total laser distance as $\mathrm{D}=8000 \mathrm{~mm}$. This is about $7 \%$ of $0.05 \mathrm{deg}$ as our adjust target, and the adjustment precision meet the requirements.

2. Considered the adjustment specification of the recording mechanism, we can get the following static adjustment parameters:

Table 1 Adjustment Parameters [deg.]

\begin{tabular}{cccc} 
& Adjust target & Constraint condition & Screw adjustable range \\
\hline Rad & $\pm 0.05 \mathrm{deg}$. & $\sqrt{\operatorname{Rad}^{2}+\text { Tan }^{2}} \leq 0.05 \mathrm{deg}$. & $140 \mathrm{deg}$. \\
Tan & $\pm 0.05 \mathrm{deg}$. & $\sqrt{\operatorname{Rad}^{2}+\text { Tan }^{2}} \leq 0.05 \mathrm{deg}$. & $80.8 \mathrm{deg}$. \\
\hline
\end{tabular}

\section{Conclusion:}

We verified the feasibility of this method in the development of recording mechanism for optical disc library. This method can satisfy the static tilt adjustment precision in the early development. At the same time, because of the high accuracy of this method, especially the precision of this method can make further enhance with the increase of the evaluation system's distance, the method can be widely used in the whole process of drive design, production, and also have a very good application in the equipment inspection and standard parts verification.

\section{References}

[1] Zhenhong Liu. Construction of Blue-ray Drive Adjustment Machine System [D]. Dalian: Dalian University of Technology, 2012.

[2] Wei xiong, Jianhua Mu, Dan Wang. Expectation for the Storage Density and the Data Rate of BD Disc [J], 2009. 
[3] Jian-she MA, Long-fa PAN, Ji-gang RU, Tian-shui ZHANG, Jun-bo CHEN. Dynamical Adjust Principle and Realization of Optical Axis Tilt Angle of DVD Traverse [J], 2004.

[4] Wei Guo. The Application Research of Traverse Base with Resin Material of BD Player [D]. Dalian: Dalian University of Technology, 2013.

[5] Zhiqiang Qu. Study on Digital Focus Control System of DVD [D]. Dalian: Dalian University of Technology, 2009. 